\title{
Sartorius Pavilion - Biomimicry as a design methodology for a parametric pavilion for the Serpentine Gallery/England
}

\author{
Elton Cristovão da Silva Lima \\ Universidade Federal de Pernambuco | Brasil | elton.cristovao@ufpe.br \\ Cristina Matsunaga \\ Universidade Federal de Pernambuco | Brasil | criismnaga@gmail.com \\ Leticia Teixeira Mendes \\ Universidade Federal de Pernambuco | Brasil | leticia.mendes@ufpe.br
}

\begin{abstract}
In order to design an ephemeral pavilion located at the Serpentine Gallery (England), an experimental design approach was developed in this paper by using biomimicry strategies associated with parametric modeling. Exploring the solution-based methodology, the analysis of the sartorius muscle anatomic features such as rotation, flexion and long shape allowed inspiring the proposal of a Sartorius Pavilion which is the object of study. The experiment was implemented throughout a parametric visual script tool resulting in a model capable of rapidly and intuitively simulating shape variations, basic structural and material attributes by modifying a set of previously defined parameters.
\end{abstract}

Keywords: Biomimicry; Bio-inspired Architecture; Sartorius Muscle; Parametric Pavilion; Serpentine Gallery.

\section{INTRODUÇÃO}

O objetivo do presente artigo é desenvolver o modelo tridimensional de um pavilhão temporário paramétrico que possibilite a simulação instantânea da forma para auxiliar o arquiteto no processo de decisão e reduzir o retrabalho. Para isso se utilizará as metodologias da biomimética, o solution-based design e o projeto paramétrico.

Assim, o processo projetual do Pavilhão Sartório parte da observação da anatomia humana para buscar soluções arquitetônicas. Atualmente, essa abordagem está relacionada ao campo da Biomimética (também conhecido como design bio-inspirado), mas o termo ganhou maior notoriedade a partir das publicações de Janine Benyus, em 1997. Benyus (2006) define a Biomimética como a disciplina que estuda as soluções existentes na natureza para inspirar soluções e processos, com o objetivo de solucionar os problemas do ser humano buscando maior sustentabilidade e eficiência dos recursos. Estudos de morfologia e forma na arquitetura são atributos comumente inspiradores e copiado dos sistemas naturais (Badarnah, 2012), isto posto, o presente trabalho busca explorar esse aspecto como princípio norteador que orientará a investigação.

De acordo com Vattam et al. (2007) e Badarnah (2012) no campo da biomimética existem duas abordagens comumente utilizadas na aplicação do design bioinspirado: a problem-based e a solution-based. A primeira se caracteriza por buscar a inspiração biológica após a identificação de um problema particular e bem definido. 0 segundo método parte da investigação do elemento biológico para solucionar um problema definido posteriormente. No projeto do pavilhão Sartório a abordagem desenvolvida foi a solution-based e a solução projetual será gerada com o auxílio do plug-in paramétrico Grasshopper no software de modelagem tridimensional Rhinoceros.

Segundo Oxman (2006), a produção arquitetônica recente experimenta uma revolução apoiada em cinco modelos paradigmáticos: a geração da forma em meio digital, a transição CAD/BIM, a concepção projetual incorporando variáveis de desempenho, a utilização de algoritmos para auxiliar na criação de formas e o projeto arquitetônico utilizando, associadamente, todos os métodos citados. Esses paradigmas se aproximam da área da Arquitetura, Engenharia, Construção e Operação; práticas de projeto já incorporadas e utilizadas nas indústrias automobilística aeronáutica e naval, como projeto integrado (AsBEA,2013).

Sendo assim, o projeto paramétrico tem ganhado espaço no campo da arquitetura e construção por colaborar com o desenvolvimento e exploração de formas arquitetônicas, além de possibilitar a experimentação de conceitos não tão utilizados no processo de projeto de arquitetura.

Tedeschi (2014) ressalta que as pesquisas acadêmicas mais recentes buscam inovar tentando escapar das limitações de edição nas quais se utilizam largamente as 
ferramentas CAD, e buscam possibilitar outras formas de manipulação destes softwares através da programação.

Muitos projetistas logo perceberam que programas mais sofisticados poderiam controlar a complexidade da forma além da capacidade humana através da estruturação de hábitos e procedimentos. Esse tipo de modelagem depende de linguagens de programação que expressam instruções de forma que um computador possa executar através de um processo sequenciado: o algoritmo (Tedeschi, 2014 - traduzido pelos autores).

O algoritmo permite direcionar a solução para uma pergunta ou executar uma determinada tarefa através de uma lista finita de instruções básicas e bem definidas (Tedeschi, 2014). Dessa forma os algoritmos acompanham a aptidão humana no processo de divisão de um problema em pequenas etapas que facilmente podem ser processadas. Apesar de ser associado à computação, os algoritmos também podem ser definidos sem necessidade das linguagens de programação.

Com o expressivo desenvolvimento tecnológico, as teorias e processos de projeto têm se reformulado. Atualmente, as metodologias de design paramétrico são capazes de se adaptar às mudanças de contexto devido as influências de linguagens paramétricas e processos generativos (Woodbury, 2010)

Dino (2012) discute que o processo generativo requer 4 elementos. O input é o responsável pela entrada de dados, ou seja, as condições iniciais e parâmetros. O output é por onde ocorre a saída de dados e geração de variantes. Além destes, é necessário um mecanismo generativo (regras, algoritmos etc.) e a seleção da melhor variante. Dessa forma, compreende-se um sistema generativo como um sistema de produção e não apenas uma construção representacional, em que o arquiteto é um ator ativo no processo projetual.

Sendo assim, o método paramétrico consiste em definir relações topológicas entre as partes de um objeto arquitetônico antes do dimensionamento. Em geral, as dimensões não são valores fixos, mas podem ser intervalos numéricos, relacionados às medidas dos componentes construtivos, resultando em maior flexibilidade e variedade de forma (Monedero, 2000). Uma das vantagens da parametrização é que quando bem utilizada permite economizar tempo e ser útil no gerenciamento do processo de projeto por permitir, de modo interativo, alterar características de um objeto (Monedero, 2000).

Dentro desse contexto, a forma do objeto arquitetônico surge a partir de regras e restrições bem definidas, que possuem coerência com os parâmetros previamente estabelecidos e incorporados a ferramentas de desenho digitais, associando, assim, a manipulação da forma com a programação (Tedeschi, 2014).

A biomimética unida ao projeto paramétrico aplicado à arquitetura é uma metodologia emergente na atualidade. Arquitetos renomados do mundo inteiro tiveram a oportunidade de testar novas soluções arquitetônicas no Serpentine Gallery Pavilion em Londres, desde sua primeira edição em 2000 (Figura 1 e 2). As exposições do
Serpentine Gallery servem como um canteiro no qual os arquitetos podem testar e explorar os limites da arquitetura experimental quanto a aplicação de processos criativos, materiais e métodos construtivos alternativos ou pouco difundidos (Tunçbilek, 2013). Nesse contexto, a exposição conta com uma equipe de engenharia multidisciplinar que se comprometem em viabilizar as obras as propostas dos pavilhões.

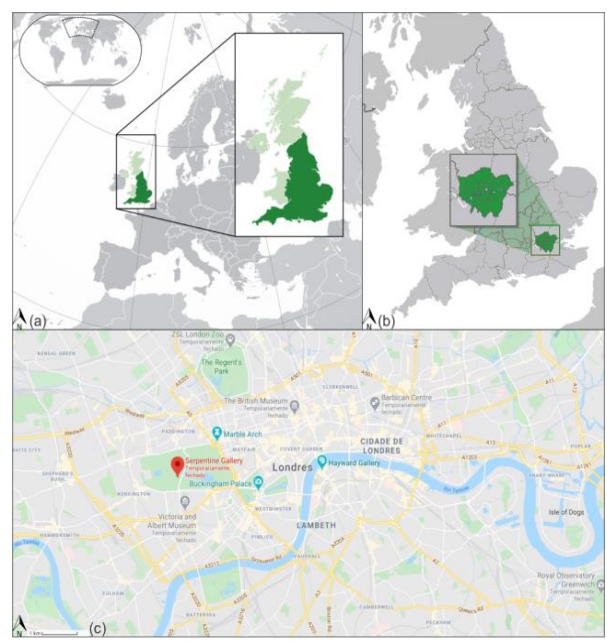

Figura 1: Localização da Serpentine Gallery em Londres Inglaterra - Reino Unido - Europa (adaptação de Wikipedia, 20-; Google Earth, 2020).

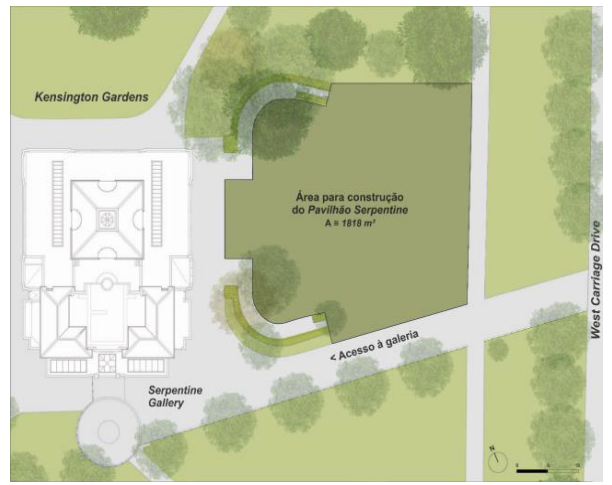

Figura 2: Localização da área destinada ao Pavilhão no jardim da Serpentine Gallery, em Kensington Gardens, em Londres (adaptação de Eumiesaward, 2005).

Dessa forma, tendo em vista o contexto internacional onde a exposição Serpentine Gallery Pavilion se destaca, o local foi escolhido como sítio para inserção do Pavilhão Sartório, já que dispõe de uma estrutura adequada para a implantação deste tipo de equipamento arquitetônico.

\section{METODOLOGIA}

Com o propósito de propor soluções sustentáveis para os desafios humanos, tem sido buscado emular a natureza, e como forma de direcionar o pensamento estratégico para este fim, a literatura aborda algumas estratégias, dentre as 
quais a denominada solution based (baseada na solução). Esta abordagem, discutida por Badarnah (2012) e Vattam et al (2007), requer que primeiramente se encontre uma estratégia na natureza, ou seja, um elemento natural inspirador que pode ser utilizado para solucionar problemas relacionados às necessidades humanas.

Por conseguinte, partiu-se para uma análise mais detalhada do elemento natural. O músculo Sartório também é conhecido como músculo costureiro, como referência à posição que os alfaiates se sentavam com as pernas cruzadas. Este músculo é o mais longo do corpo humano, geralmente excedendo $50 \mathrm{~cm}$ de comprimento e percorrendo e movimentando as articulações do quadril e joelho - por isso é considerado um músculo biarticular (Gray, 1918). O músculo Sartório é estreito, em forma de fita e atua flexionando, abduzindo (movimento no plano frontal, quando um membro se move para longe da linha média do corpo) fracamente e rotacionando lateralmente a coxa; já no joelho, o músculo é responsável por flexionar e rotacionar medialmente a perna (Dziedzic, Bogacka \& Ciszek; 2014) (Figura 3 e 4).

Após o estudo anatômico do músculo Sartório, foi possível estabelecer uma analogia morfofuncional (Soares \& Arruda, 2017). Assim, foram explorados tanto os atributos formais e estruturais do músculo sartório como sua forma estreita e alongada, e sua posição de inserção; quanto às funções e qualidades específicas como as ações de flexão e rotação.

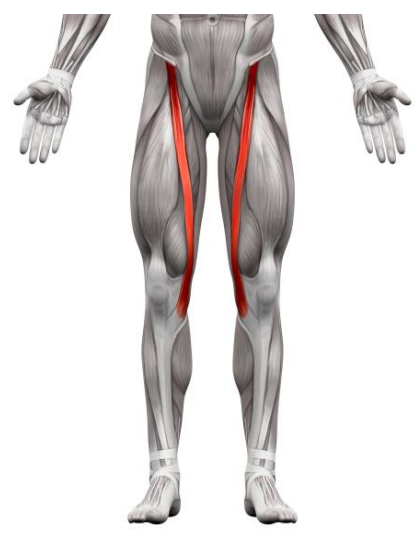

Figura 3: Vista frontal do músculo sartório (Istock, 2016).

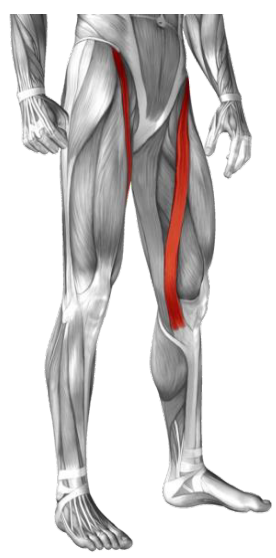

Figura 4: Vista lateral dos membros inferiores do corpo humano com destaque para o músculo Sartório (Stockett, 2018).

Diante de todas as características relevantes levantadas a partir do músculo sartório foi possível o avanço para a fase de modelagem paramétrica através da implementação de um código no Grasshopper [1]. A utilização de uma ferramenta de script visual permitiu fazer testes morfológicos e de condicionantes com o intuito de respeitar parâmetros estabelecidos por pavilhões anteriores, como área e altura por exemplo. A partir de alterações feitas nos parâmetros do código implementado, foi possível obter diversos resultados morfológicos respeitando as características bio-inspiradas.

\section{RESULTADOS}

A partir do estudo anatômico do músculo Sartório, buscouse desenvolver um pavilhão com dois tipos de estruturas que refletissem a morfologia alongada e os aspectos de flexibilidade e rotação (Figuras 5 a 8).

A estrutura central, e principal, em forma de trapézio alongado e sustentado por arcos de madeira lamelada colada (MLC), coberta com peças longas, também em MLC, em formato de fita que rotacionam em graus diferentes e são dispostas ao longo dos arcos (Figura 2). Este tipo de material consiste na colagem de finas lâminas de madeira dispostas de modo que suas fibras fiquem paralelas entre si, e o aspecto estrutural se dá a partir do uso de colas de alta resistência. A MLC é facilmente maleável, resistente, proporciona flexibilidade com curvaturas, formas arqueadas e dobradas, bem como permite grande envergadura (Migliani, 2019). Assim percebe-se que a escolha do material também permite a associação com as características anatômicas do músculo Sartório.

As estruturas laterais, secundárias, são metálicas, cobertas por painéis de vidro e sustentadas por arcos alongados dispostos em uma inclinação que permite que a maior parte fique sob a estrutura principal (Figura 6). 


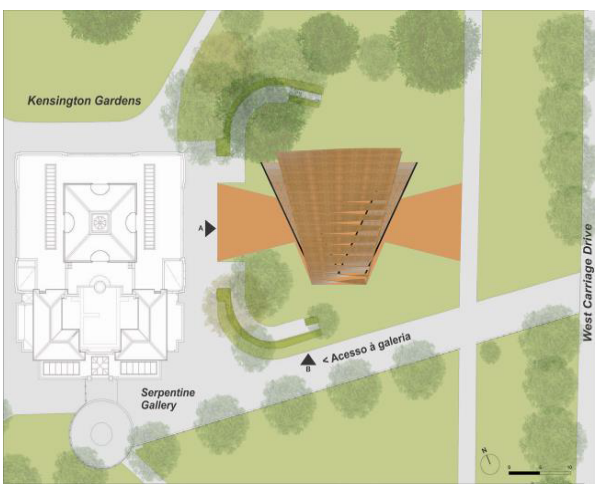

Figura 5: Vista Superior do Pavilhão Sartório (adaptação de Eumiesaward, 2005).

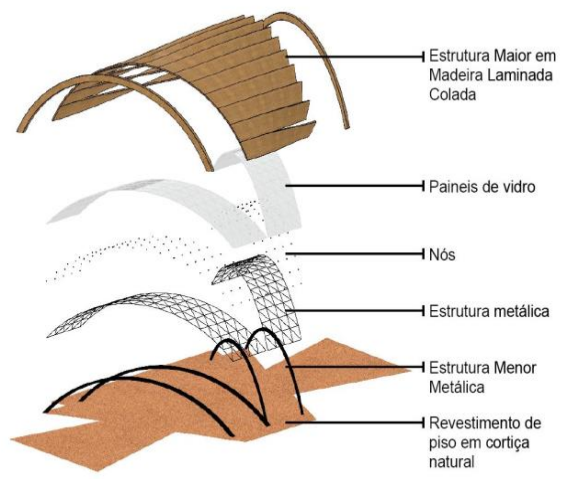

Figura 6: Perspectiva explodida da estrutura do pavilhão Sartório.

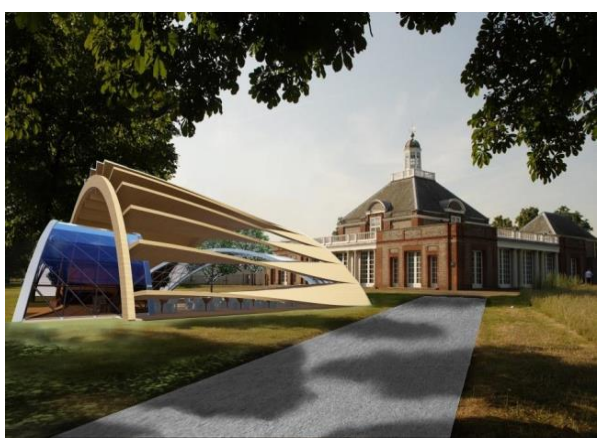

Figura 7: Perspectiva externa do pavilhão Sartório.

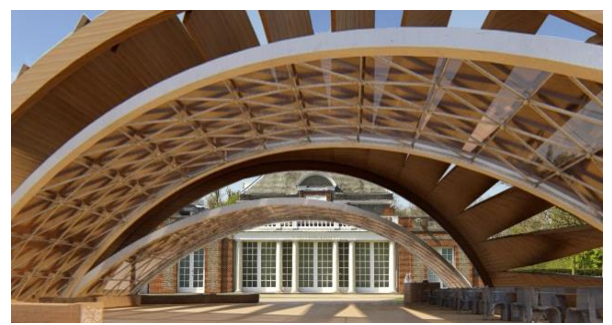

Figura 8: Perspectiva interna do pavilhão Sartório.

O desenvolvimento do projeto do pavilhão levou em consideração as edições anteriores da exposição na Serpentine Gallery quanto aos aspectos de área, altura e materiais, sendo possível modificar estes parâmetros dentro do código implementado para fazer testes. Com isso, através do mesmo código foi possível explorar e gerar diferentes soluções projetuais considerando o mesmo contexto.

O código desenvolvido permitiu parametrizar todo 0 processo de projeto como espessuras e alturas das estruturas, dimensionamento de painéis, grau de rotação das placas de madeira que cobrem a estrutura principal, entre outros detalhes técnicos. No entanto, para a finalidade de geração de diversas soluções, é importante destacar que não foram levados em consideração cálculos estruturais precisos, sendo necessário uma avaliação por uma equipe especialista, como ocorre normalmente nos projetos dos pavilhões Serpentine. A partir disso, foi possível simular várias formas dentro dos parâmetros prédefinidos, das quais quatro serão documentadas nesse artigo e se destacaram por retratar variações extremas da forma dentro das restrições impostas (Figura 9).

Todos os itens listados na ficha técnica (Figura 9) são passiveis de variações e foram parâmetros considerados na implementação do código no Grasshopper. A tabela sintetiza essa variabilidade e mostra quatro opções morfológicas do pavilhão Sartório que foram geradas de modo interativo já que o código é responsivo e permite que várias possibilidades sejam testadas de forma ágil.

Mudanças de parâmetros como inclinação dos arcos da estrutura de madeira, ou posicionamento da estrutura metálica podem gerar resultados diferentes, como os pavilhões 1 e 3, por exemplo. O experimento 01 se caracteriza por arcos de madeira cujo raio varia gradualmente assim como os painéis de vidro, o que atribui forma mais dinâmica. Em contraste, o experimento 3 apresenta forma mais retilínea tanto dos painéis de vidro como da estrutura em arcos de madeira, o que a torna mais previsível. Já quanto a altura e área de implantação percebe-se que os pavilhões 2 e 4 são bem divergentes, essa diferença ocorre devido ao posicionamento dos pontos de inserção de cada estrutura e afeta diretamente tanto na quantidade de placas de madeira que o recobrem quanto na estruturação dos painéis de vidro, por exemplo. A variabilidade de altura no pavilhão Sartório foi importante para auxiliar no estudo da relação do pavilhão com o a galeria Serpentine, assim como na definição das visadas mais interessantes na altura do observador. 


\begin{tabular}{|c|c|c|c|c|}
\hline \multicolumn{5}{|c|}{ Tabela de parâmetros e restrições do Pavilhão Sartório } \\
\hline & Experimento 1 & Experimento 2 & Experimento 3 & Experimento 4 \\
\hline \multicolumn{5}{|l|}{ Perspectiva } \\
\hline \multicolumn{5}{|l|}{ Vsita superior } \\
\hline \multicolumn{5}{|l|}{ Vistas laterais } \\
\hline Área $\left(m^{2}\right)$ & 469,64 & 545,91 & 440 & 814 \\
\hline $\begin{array}{l}\text { Altura (m) Estrutura } \\
\text { Maior }\end{array}$ & 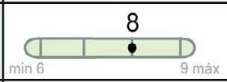 & 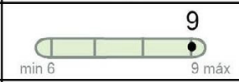 & 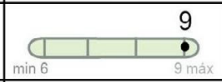 & 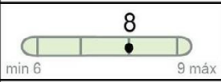 \\
\hline $\begin{array}{l}\text { Altura (m) Estrutura } \\
\text { Menor }\end{array}$ & 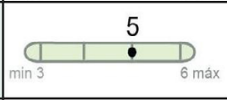 & 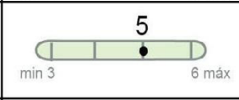 & 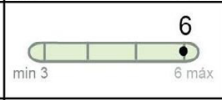 & 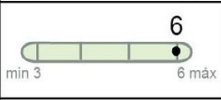 \\
\hline $\begin{array}{l}\text { Quantidade de Placas de } \\
\text { Madeira }\end{array}$ & 15 & 12 & 16 & 18 \\
\hline $\begin{array}{l}\text { Espessura das Placas } \\
\text { de Madeira }(\mathrm{cm})\end{array}$ & 15 & 15 & 15 & 15 \\
\hline $\begin{array}{l}\text { Arcos Laterais - Madeira } \\
\text { (altura } \mathrm{H} \text {, largura) }(\mathrm{cm})\end{array}$ & 70,30 & 70,30 & 50,30 & 90,30 \\
\hline $\begin{array}{l}\text { Arcos Laterais - } \\
\text { Metálicos (altura } \mathrm{H}, \\
\text { largura) (cm) }\end{array}$ & 30,30 & 30,20 & 40,30 & 40,30 \\
\hline \multicolumn{5}{|c|}{ Estrutura Metálica (os perfis circulares podem ser trocados por retangulares) } \\
\hline 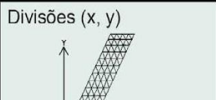 & $(4,15)$ & $(4,15)$ & $(4,16)$ & $(5,18)$ \\
\hline$\emptyset$ Perfis Metálicos $(\mathrm{cm})$ & 6 & 6 & 6 & 6 \\
\hline \multicolumn{5}{|c|}{ Painéis de Vidro (painéis com medidas distintas, necessário fazer um mapa de peças) } \\
\hline 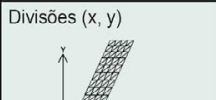 & $(4,15)$ & $(4,15)$ & $(4,16)$ & $(5,18)$ \\
\hline Quantidade Vidro $\left(\mathrm{m}^{2}\right)$ & 226,5 & 204,06 & 271,74 & 611 \\
\hline
\end{tabular}

Figura 9: Ficha técnica dos parâmetros implementados na fase de modelagem paramétrica. 


\section{DISCUSSÃO}

O fato de os pavilhões da Serpentine Gallery serem um tipo de laboratório de experimentação e ao mesmo tempo espaços públicos e de eventos dá oportunidade a arquitetos de exporem seus projetos e metodologias de trabalho. A construção do pavilhão envolve uma equipe multidisciplinar para atender as várias disciplinas projetuais essenciais; ARUP e AECOM [2], por exemplo, são empresas de engenharia que colaboraram para os projetos e construções dos pavilhões desde sua primeira edição, em 2000.

A biomimética por trás da construção justifica as decisões projetuais e enriquece o projeto final a partir de um embasamento conceitual trazido da natureza e refere-se a uma área da ciência que não seria facilmente relacionada com a arquitetura e construção civil.

Nesta pesquisa, a anatomia humana foi a área da biologia onde a metodologia da biomimética se debruçou, por entender-se que o homem também faz parte da natureza e, portanto, todos os elementos que o constituem são passíveis de estudos e descobertas favoráveis para inspirar um projeto. A anatomia humana é farta de detalhes visíveis e invisíveis à olho nu, das células aos maiores órgãos, os quais muitos podem servir de inspiração. Bardanah (2012) reconhece que um dos desafios do design bio-inspirado é selecionar o elemento natural diante do enorme banco de dados que caracteriza a natureza e também ajustar a escala que pode ser tanto investigada a nível microscópico, como a olho nu, por exemplo. Isso demonstra uma tendência multidisciplinar no processo projetual (Vattam et al, 2007) já em seu desenvolvimento existe a cooperação de profissionais de diferentes áreas; assim como é comum o auxílio de programadores no design generativo, profissionais da área da biologia trabalham eficazmente com designers e arquitetos.

A modelagem paramétrica exigiu o uso de ferramentas adicionais para geração da morfologia do Pavilhão Sartório, os plug-in's, disponíveis para uso dentro do Grasshopper. A ferramenta Pufferfish [3] foi útil para fornecer e controlar a espessura das estruturas de madeira em forma de fita que compreendem a cobertura de formato trapezoidal, além de simplificar a realização de operações com curvas. O Lunchbox [4] permitiu criar a malha metálica treliçada e os painéis triangulares que integram a cobertura das estruturas secundárias de arcos menores. O Human [5], simplificou o trabalho com listas e possibilitou selecionar objetos específicos de maneira mais objetiva. Por fim, o Heteroptera [6], atuou facilitando a construção básica da parte geométrica através de componentes matemáticos simples.

A criação de uma geometria variacional está diretamente ligada ao conceito de modelos paramétricos que permitem a interação e edição do modelo em qualquer etapa da implementação, sendo necessário apenas a modificação de alguns parâmetros como dimensões e restrições, por exemplo. Esse processo dispensa o processo manual de edição da forma, permite a adição de novos parâmetros sem comprometer a visualização instantânea do objeto arquitetônico.

Ademais, o projeto paramétrico facilita para o arquiteto inovar, explorar e testar diversas formas visto que consegue variados resultados de forma automática, com apenas a variação de alguns parâmetros, otimizando assim o processo de projeto.

\section{CONCLUSÃO}

Diante da motivação de reduzir o retrabalho e explorar a interatividade do modelo tridimensional paramétrico, sob a ótica da biomimética e do design paramétrico, foi desenvolvido o pavilhão sartório.

A partir do processo de projeto, buscou-se analisar a viabilidade da integração entre a biomimética e a modelagem paramétrica. Enquanto a primeira é constituída de conceitos e abstração - é nesta fase que surge a ideia que fundamenta o projeto a partir da inferência de dados e padrões encontrados na natureza, a segunda permite materializar as ideias e fornecer a visualização da forma final de maneira ágil a partir da mudança de parâmetros pré-estabelecidos.

Desde o início sabia-se do intuito de projetar o pavilhão, mas partir de um elemento natural foi um desafio devido a imensa quantidade de reinos e espécies (entre outras classificações) disponíveis para investigação na natureza. Então, para restringir a busca por uma inspiração, o corpo humano tornou-se um elemento de estudo já que a anatomia humana é uma área ampla e apresenta grande potencial de aplicação no processo projetual, como explorado por diversos arquitetos. Nesta exploração, o músculo Sartório apresentou características expressivas pelos seus atributos morfológicos e funcionais. Ele aparentemente se torce para se inserir adequadamente da anatomia da coxa, e isso mostrou-se como uma possibilidade de imitação que poderia ser representada através de formas matemáticas e assim transferi-las para o campo da arquitetura por meio de uma estrutura com características semelhantes.

A metodologia solution-based só foi aplicada após a definição do elemento natural, neste caso o músculo Sartório. Assim, foi possível alcançar a variabilidade de forma paramétrica, que contribui para visualização da solução e agiliza o processo de avaliação da forma pelo projetista. No processo de projeto tradicional a avaliação para definição de uma solução gerava uma quantidade excessiva de remodelagens e reconstrução de geometrias, para testar variáveis diversas. No projeto paramétrico, como proposto nesse artigo, algumas dessas variáveis como altura mínima ou máxima, disposição e espessura da estrutura do pavilhão são relacionadas a valores variáveis e regras que permitem que o modelo se torne dinâmico e responsivo. Isso sinaliza uma mudança de paradigma, no qual o arquiteto tende a concentrar seus esforços mais no processo de design em relação ao resultado final.

No caso do Pavilhão Sartório um dos desdobramentos futuros poderia consistir na incorporação de plug-ins como o Karamba [7] e o Kangaroo [8] para avaliação e análise estrutural, bem como a utilização da ferramenta Ladybug [9] para simulações de performance climática. Adicionalmente, também seria interessante como desdobramento futuro a incorporação de parâmetros de análise mais subjetivos como, orientação da visada ou altura ideal em relação as construções do entorno. 
As limitações da prática projetual existentes no exercício prática da arquitetura foram uma das grandes motivações desse artigo. As abordagens da Biomimética e do projeto paramétrico incitam métodos alternativos na tentativa de obter resultados formais mais expressivos e que, além disso, reduza a necessidade de retrabalho da modelagem das soluções no processo de concepção do projeto e tomada de decisão. A partir do que foi desenvolvido nesse artigo foi possível observar que as abordagens projetuais utilizadas se complementam e que a complexidade do processo de concepção arquitetônica tende a aumentar a medida que outras disciplinas como a biologia ou a programação sejam utilizadas como input ou ferramenta para o desenvolvimento do projeto.

\section{NOTAS}

[1] O Grasshopper é uma ferramenta que opera com algoritmos generativos de modelagem associativa de script visual, ou seja, é uma linguagem de programação visual capaz de construir geometrias a partir da conexão de componentes e parâmetros (Brod, Pires \& Borba; 2012).

[2] Tanto a ARUP e a AECOM são empresas multinacionais que oferecem serviços de engenharia, arquitetura e gerenciamento. Elas têm participação importante no desenvolvimento dos pavilhões da Serpentine Gallery, sendo responsável pelo projeto estrutural.

[3] O plug-in Pufferfish consiste em um conjunto de ferramentas com foco em operações para mudanças de forma, como transformações e interpolações, por exemplo (Parametric House, 2020).

[4] A ferramenta Lunchbox consiste em um conjunto de componentes de design computacional utilizado para explorar formas matemáticas, painéis, estruturas e fluxo de trabalho (Food4Rhino, 2018).

[5] O plug-in Human é utilizado para criar e referenciar geometrias, incluindo luzes, blocos e objetos de texto (TEER, 2016).

[6] O Heteroptera funciona como uma caixa de ferramentas, adicionada à interface do Grasshopper, que inclui componentes de animação, matemática, networks e probabilidade (BAHRAMI, 2017).

[7] O plug-in Karamba permite desenvolver e fazer dimensionamento estrutural por trabalhar com análise precisa de estruturas (Karamba3D, 2020).

[8] A ferramenta Kangaroo consiste em um conjunto de componentes úteis para fazer simulações físicas, encontrar formas e solucionar restrições geométricas (MEIER, 2020).

[9] O plug-in Ladybug analisa a incidência solar sobre um objeto e fornece gráficos interativos que mostram o caminho do sol, rosa dos ventos e estudo de sombras, por exemplo (Foof4Rhino, 2020).

\section{REFERÊNCIAS}

AsBEA. (2013. Guia AsBEA Boas Práticas em BIM: Estruturação do Escritório de Projeto para a Implantação do BIM. Fascículo I. São Paulo.

Badarnah, L. (2012). Towards the living envelope: Biomimicry for building envelope adaptation. Delft University of Technology.

Bahrami, A. (2017). Heteroptera. Retrieved from https://grasshopper3d.com/group/heteroptera?overrid e

Benyus, J. (2006). Biomimicry: Innovation inspired by nature. Harper Perennial, New York.

Brod, G. A., Pires, J., \& Borda, A. (2012). Um ensaio para inserção do conceito de processos generativos digitais em estágios iniciais da formação em arquitetura [An experiment for introducing the concept of generative processes in early stages of architectural education].

Dziedzic, D., Bogacka, U. \& Ciszek, B. (2014). Anatomy of the sartorius muscle. Folia Mophol 73:259-262. Retrieved from https://journals.viamedica.pl/folia_morphological/article /view/FM.2014.0037/34236

Eumiesaward. (2005). Serpentine Gallery Pavillion 2005 (dismantled). Retrieved from https://www.miesarch.com/work/1187

Fisher, T., Herr, C. M. (2001). Teaching Generative Design. In: Proceedings of the 4th International Generative Art Conference, Generative Design Lab DIAP, Politecnico di Milano. Retrieved from https://www.generativeart.com

Food4rhino. (2018). Lunchbox. Retrieved from https://https://www.food4rhino.com/app/lunchbox

Food4rhino. (2020). Ladybug tools. Retrieved from https://www.food4rhino.com/app/ladybug-tools

Gray, H. (1918). Anatomy of the Human Body. Philadelphia: Lea \& Febiger. Bartleby.com, 2000. Retrieved from https://www.bartleby.com/107/

Google Earth. (2020). Retrieved from: google.com.br/maps

Gürsel Dino, I. (2012). Creative design exploration by parametric generative systems in architecture, METU JFA, 29(1), 207-224.

Istock. (2016). Sartorius Muscle - Anatomy Muscles isolated on white. Retrieved from https://www.istockphoto.com/br/foto/sart\%C3\%B3rio$\mathrm{m} \% \mathrm{C} 3 \%$ BAsculos-anatomia-muscular-isolado-nobranco-gm533846530-94583675

Karamba. (2020). Karamba3D. Retrieved from https://www.karamba3d.com/

Light, R., Gossard, D. (1981). Variational Geometry in CAD, Computer Graphics, 15 (3), 1712-1777.

Meier, M. (2016). Kangaroo Physics Simulation for Grasshopper. Retrieved from https://mkmra2.blogspot.com/2016/11/kangaroophysics-simulation-for.html 
Migliani, A. (2019). O que é Madeira Colada (MLC ou Glulam)? ArchDaily Brasil. Retrieved from https://www.archdaily.com.br/br/928061/o-que-emadeira-laminada-colada-mlc-ou-glulam ISSN 07198906

Monedero, J. (2000). Parametric design: a review and some experiences. Automation in Construction, v. 9, n. 4, 369-377.

Oxman, R. (2006). Theory and design in the first digital age. Design Studies, v. 27, n. 3, p. 229-265.

Parametric House. (2020). Pufferfish. Retrieved from https://parametrichouse.com/pufferfish/

Soares, T., Arruda, A. (2017). Ecomateriais biomiméticos, um caminho eficiente para a sustentabilidade. Mis Sustentável, Florianópolis, v. 3, n. 4, 29-45.

Stockett, S. (2018). Sartorius: Learn Your Muscles. Retrieved https://www.custompilatesandyoga.com/sartoriuslearn-your-muscles/

Teer, M. (2016). New to the human plugin? Retrieved from https:// blackspectacles.com/blog/post/extra-featuresin-human-free-

tutorial\#: :text=Human $\% 20$ is $\% 20 a \% 20$ plugin $\% 20$ that, $\% 2 \mathrm{C} \% 20$ linetypes $\% 2 \mathrm{C} \% 20$ and $\% 20$ other $\% 20$ settings
Tedeschi, A. (2014). AAD, Algorithms-Aided Design: Parametric Strategies using Grasshopper. 1. Ed. Brienza: Le Penseur.

Tunçbilek, G. (2013). Temporary Architecture: The Serpentine Gallery Pavilions. Master Thesis. Turkey: Middle East Technical University.

Vattam, S., Helms, M., and Goel, A.K., [2007]. Biologically Inspired Innovation in Engineering Design: A Cognitive Study. Technical Report, Graphics, Visualization and Usability Center, Georgia Institute of Technology, GITGVU-07-07.

Wikipedia English. (20--). England. Retrieved from: https://en.wikipediaa.org/wiki/England

Woodbury, R. (2010). Elements of Parametric Design. Routledge, NY. 